\title{
In Vivo Toxicity Assessment of Gold Nanoparticles in Drosophila melanogaster
}

\author{
Pier Paolo Pompa ${ }^{1}(\varangle)$, Giuseppe Vecchio ${ }^{1}$, Antonio Galeone ${ }^{1}$, Virgilio Brunetti ${ }^{1}$, Stefania Sabella ${ }^{1}$, Gabriele \\ Maiorano $^{1}$, Andrea Falqui ${ }^{2}$, Giovanni Bertoni ${ }^{2}$, and Roberto Cingolani ${ }^{1,2}$ \\ ${ }^{1}$ Italian Institute of Technology, Center for Bio-Molecular Nanotechnology, Via Barsanti, 1-73010 Arnesano (Lecce), Italy \\ ${ }^{2}$ Italian Institute of Technology, Central Research Laboratories, Via Morego, 30-16136 Genova, Italy \\ Received: 29 October 2010 / Revised: 17 December 2010 / Accepted: 17 December 2010 \\ (C) Tsinghua University Press and Springer-Verlag Berlin Heidelberg 2011
}

\begin{abstract}
The growing use of nanomaterials in commercial goods and novel technologies is generating increasing questions about possible risks for human health and environment, due to the lack of an in-depth assessment of their potential toxicity. In this context, we investigated the effects of citrate-capped gold nanoparticles (AuNPs) on the model system Drosophila melanogaster upon ingestion. We observed a significant in vivo toxicity of AuNPs, which elicited clear adverse effects in treated organisms, such as a strong reduction of their life span and fertility, presence of DNA fragmentation, as well as a significant overexpression of the stress proteins. Transmission electron microscopy demonstrated the localization of the nanoparticles in tissues of Drosophila. The experimental evidence of high in vivo toxicity of a nanoscale material, which is widely considered to be safe and biocompatible in its bulk form, opens up important questions in many fields, including nanomedicine, material science, health, drug delivery and risk assessment.
\end{abstract}

\section{KEYWORDS}

Nanoparticles, nanotoxicology, gold, in vivo studies

\section{Introduction}

The well established concept of biocompatibility is recently experiencing severe limitations, due to the large advent of nanoscale materials, and a new field, known as nanotoxicology, is strongly emerging [1-5]. Several nanomaterials, such as carbon nanotubes, quantum dots, metal and metal-oxide nanoparticles, have been recently observed to show significant toxicity [6-9], thus introducing the general perception of a possible size dependence of material toxicity $[1,10]$. In this regard, gold is typically considered as one of the most inert and biocompatible materials and, even at the nanoscale, several in vitro investigations have suggested that it is relatively safe, although some recent papers have also reported adverse effects, especially in the case of very small gold clusters [11-19]. In this work, however, we show that nanoscale gold is highly toxic in vivo. We studied the effects of $15 \mathrm{~nm}$ citratecapped gold nanoparticles (AuNPs) on the model system Drosophila melanogaster upon ingestion, finding dramatic consequences for the organism life span and fertility. Moreover, we also observed that AuNPs elicit DNA damage along with a clear biochemical response in the organisms, which exhibit a significant overexpression of the stress proteins upon treatment.

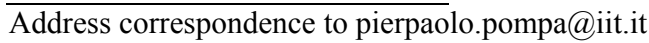




\section{Experimental}

\subsection{AuNP synthesis}

$15 \mathrm{~nm}$ sodium citrate-capped AuNPs were synthesized based on colloidal solutions according to the classical Turkevich-Frens method [20,21], using trisodium citrate as reducing agent in aqueous solution. All glassware and the magnetic stir-bars were washed thoroughly with aqua regia $\left(\mathrm{HCl}\right.$ and $\mathrm{HNO}_{3}$ in a 3:1 volumetric ratio). Briefly, $150 \mathrm{~mL}$ of a $0.25 \mathrm{mmol} / \mathrm{L}$ aqueous solution of $\mathrm{HAuCl}_{4}$ (Sigma-Aldrich) in a threenecked flask, with a condenser and a thermometer, was heated to boiling with a heating mantle while stirring. After boiling had commenced, $2.9 \mathrm{~mL}$ of a $38.8 \mathrm{mmol} / \mathrm{L}$ aqueous solution of sodium citrate (Sigma-Aldrich) was added to give a citrate/ $\mathrm{HAuCl}_{4}$ ratio of 3.10:1. The solution changed color rapidly in the following sequence: pale yellow, colorless, very dark blue, purple, and finally wine red. The solution was kept gently boiling for $30 \mathrm{~min}$ and then slowly cooled down. To minimize the influences of solvent and unreacted reagents in the toxicology studies, the solution was then immediately centrifuged at $12000 \mathrm{~g}$ (relative centrifugal force) for $15 \mathrm{~min}$ to isolate the particles, which were resuspended in $150 \mathrm{~mL}$ of ultrapure water. This procedure was repeated three times. The resulting particles were coated with negatively charged citrate and were, hence, well-suspended (Fig. 1). The $\mathrm{pH}$ of the AuNP solution was measured to be 7.4. Details of characterization of the AuNPs are given in Fig. S-1 and the associated text in the Electronic Supplementary Material (ESM).

\subsection{Drosophila melanogaster strain and culture conditions}

The flies and larvae of wild-type Drosophila melanogaster (Oregon $\mathrm{R}+$ ) were cultured at $24^{\circ} \mathrm{C} \pm 1{ }^{\circ} \mathrm{C}$ on standard Drosophila food, containing agar, corn meal, sugar, yeast and nepagin (methyl-p-hydroxybenzoate).

\subsection{Treatment of Drosophila with AuNPs}

For toxicity assays, AuNPs were formulated in the diet. Six different concentrations $(1.9,3.8,19,38,190$, and $380 \mathrm{pmol} / \mathrm{L}$ ) of AuNPs dispersed in the food were used for experiments. The $380 \mathrm{pmol} / \mathrm{L}$ concentration of

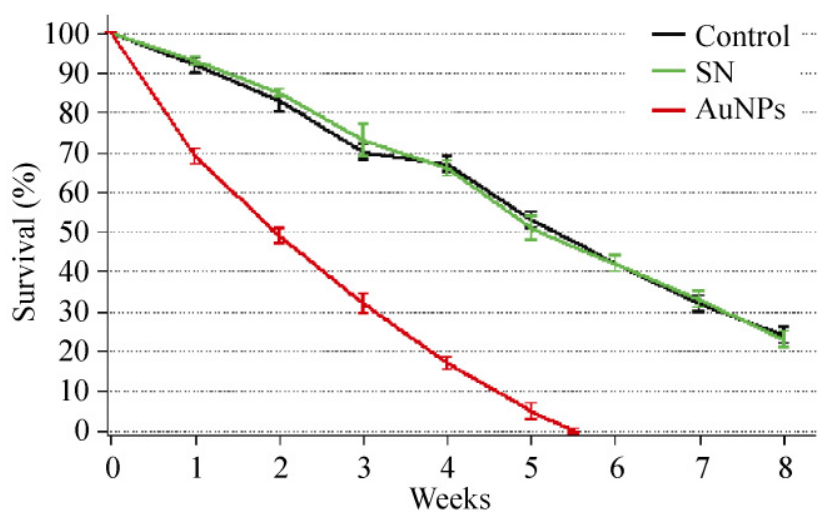

Figure 1 Lifespan curves of Drosophila flies nurtured with AuNP-treated food (380 pmol/L) (AuNPs, red line) as compared to two populations bred with normal food (Control, black line) or supernatant-treated food ( $\mathrm{SN}$, green line). Experimental points represent the average from 5 independent experiments and the error bars indicate the standard deviation. To assess statistical significance of survival curves, Student's $t$-test was applied giving $p<0.01$ (AuNP treatment compared to the control), $p<0.01$ (AuNPs compared to the $\mathrm{SN}$ ), $p>0.05$ (control compared to the $\mathrm{SN}$ ). The survival distributions were also evaluated by non-parametric Mantel-Cox test giving $p<0.05$ (AuNP treatment with respect to the control; log-rank $\chi^{2}=5.278, \mathrm{df}=1$ ), $p<0.05$ (AuNP treatment with respect to $\mathrm{SN} ; \chi^{2}=4.427, \mathrm{df}=1$ ) and $p>0.05$ (SN with respect to control; $\chi^{2}=0.3207, \mathrm{df}=1$ ). The comparison of the half-lives of treated vs. control population gives $p<0.001$

AuNPs corresponds to a $39.3 \mu \mathrm{mol} / \mathrm{L}$ concentration of $\mathrm{Au}$ (the conversion factor was obtained by calculating that one $15 \mathrm{~nm}$ AuNP contains $1.04 \times 10^{5} \mathrm{Au}$ atoms). Assuming that Drosophila typically eats $1.5 \mu \mathrm{L} /$ day of food [22], the maximum dose of AuNPs administered to the flies ( $380 \mathrm{pmol} / \mathrm{L}$ AuNPs) is $12 \mu \mathrm{g} / \mathrm{g}$ per day. In particular, the solution containing AuNPs was added to the food before solidification, mixed strongly and finally poured into vials. TEM analyses showed that the AuNPs do not significantly aggregate after mixing with the Drosophila food, maintaining a good degree of monodispersity. With the same modality, we prepared food with the AuNP supernatant (SN), obtained by centrifugation of the AuNP solution at $13400 \mathrm{rpm}$ for $30 \mathrm{~min}$. This preparation was used to exclude the presence of toxic compounds in the solution containing AuNPs.

\subsection{Lifespan experiments}

For longevity analyses, flies were maintained in vials in groups of 10 ( 5 females and 5 males) and transferred 
into new vials every 4 days. We performed five independent experiments, using 100 newly eclosed flies for each experiment. In particular, we carried out this experiment using normal food, treated food containing AuNP SN and treated food containing $380 \mathrm{pM}$ of AuNPs.

\subsection{Fertility and reproductive performance}

The method of Gayathri and Krishnamurthy [23] was followed with some modifications. Virgin flies emerging from control and AuNP-treated food (with different concentrations) were isolated and pair mated in normal food vials. Pair mating was conducted in two different conditions per treatment group (30 pairs of flies were taken per each treatment group): (1) treated males with normal females; and (2) normal males with treated females. Flies were transferred into fresh vials every day for the subsequent ten days. The number of eggs laid during these ten days was scored. From the data obtained, the total fecundity (number of eggs) and mean egg production by a female for ten days were calculated. The total number of flies eclosed from the eggs laid during these ten days of pair mating was counted. The mean number of flies emerged per pair for ten days gave a measure of the reproductive performance.

\subsection{Terminal transferase dUTP nick-end-labeling (TUNEL) assay}

Midgut was dissected in Ringer's Buffer and fixed in $3.7 \%$ paraformaldehyde in phosphate buffer solution (PBS) for $3 \mathrm{~h}$. After washing in PBT (PBS, 0.1\% Tween 20), midgut was heated in $500 \mu \mathrm{L}$ of PBT for $5 \mathrm{~min}$ to approximately $85^{\circ} \mathrm{C}$ to enhance the efficiency of the labeling reaction. Midgut was then incubated in PBT containing $50 \mathrm{mmol} / \mathrm{L} \mathrm{NH}_{4} \mathrm{Cl}$ for $10 \mathrm{~min}$, and permeabilized in PBS-0.3\% Triton X-100 overnight. Midgut was processed by Click-iT TUNEL Alexa Fluor647 Imaging Assay (Invitrogen) according to the manufacturer's instructions. Midgut was equilibrated for $10 \mathrm{~min}$ in TdT TUNEL buffer and then added to TdT reaction cocktail, containing TdT enzyme and a modified dUTP, and incubated for $60 \mathrm{~min}$ at $37^{\circ} \mathrm{C}$. After incubation, midgut was washed twice with 3\% bovine serum albumin (BSA) in PBS for 2 min each. Subsequently, sample was incubated with Click-iT reaction cocktail and incubated for $30 \mathrm{~min}$ at room temperature, protected from light. Then, the Click-iT reaction cocktail was removed and tissue was washed with 3\% BSA in PBS for $5 \mathrm{~min}$ and 1X Hoechst 33342 solution for $15 \mathrm{~min}$ at room temperature. These samples were then characterized by confocal microscopy (Leica TCS-SP5 AOBS). Quantitative analyses of TUNEL-positive nuclei were carried out by examining 9 different midgut tissues (20 different microscopic fields each) from three independent experiments.

\subsection{Expression analysis of heat-shock-protein-70kDa genes}

Third instar larvae extracts were prepared by homogenizing larvae in groups of 10 in a cold solution of RNAlater (Sigma). heat-shock-protein-70kDa (hsp70) (GeneID: 48581) mRNA expression levels were examined by performing real-time quantitative polymerase chain reaction (PCR) to detect toxicity stress in treated and control larvae. mRNA was isolated using Dynabeads mRNA Direct Kit (Invitrogen) according to the manufacturer's instructions. The amount of mRNA in each sample was determined by measuring the optical density (OD) 260/280 ratio using a UV-vis spectrophotometer, and mRNA quality was analyzed using agarose gel electrophoresis (1.2\%). The qRT-PCR was carried out using QuantiTect primer assays for gene target and internal control (Qiagen, Valencia, CA, USA). qRT-PCR was performed using direct mRNAs in one-step reaction in a ABI 7500 thermal cycler (Applied Biosystems). For each gene we used $1 \mu \mathrm{L}$ of $200 \mathrm{ng} / \mu \mathrm{L}$ of mRNA solution mixed with $10 \mu \mathrm{L}$ of $10 \mathrm{X}$ Express Syber Green qPCR SuperMix premixed with ROX (Invitrogen), $2 \mu \mathrm{L}$ of 10X gene specific primers mix, $0.5 \mu \mathrm{L}$ of Express SuperScript Mix for one-Step Syber GreenER (Invitrogen) and $6.5 \mu \mathrm{L}$ of DEPC-treated water. Reaction conditions for all genes were: $50{ }^{\circ} \mathrm{C}$ for $5 \mathrm{~min}$ to perform cDNA synthesis and then immediately followed by PCR quantification program, repeated 40 times $\left(15 \mathrm{~s}\right.$ at $95^{\circ} \mathrm{C}, 1 \mathrm{~min}$ at $\left.60{ }^{\circ} \mathrm{C}\right)$. This program was followed by a melting curve program $\left(60-99{ }^{\circ} \mathrm{C}\right.$ with a heating rate of $0.1{ }^{\circ} \mathrm{C} / \mathrm{s}$ and continuous fluorescence measurements). Relative expression was calculated from cycle threshold values ( $\Delta \Delta \mathrm{Ct}$ method) using 
RpL32 (GeneID: 43573) ribosomal RNA expression as internal control for each sample.

\subsection{Transmission electron microscopy}

Dissection of Drosophila was performed in PBS, pH 7.4, and three different tissues were isolated: anterior midgut of three larval instar, ovaries and testes from adult flies. Freshly dissected tissues were then immediately fixed with $2.5 \%$ glutaraldehyde, and $0.1 \mathrm{~mol} / \mathrm{L}$ sodium cacodylate buffer $\mathrm{pH} 7.3$ at $4{ }^{\circ} \mathrm{C}$ for $2 \mathrm{~h}$. Subsequently, unreacted glutaraldehyde was removed by washing the samples with the buffer 2-3 times. Samples were resuspended in $1 \% \mathrm{OsO}_{4}$ in the same buffer and incubated for $2 \mathrm{~h}$, followed by two washes with the buffer. After post-fixation, dehydration was carried out once for $10 \mathrm{~min}$ in each of a graded ethanol solution series $(10 \%, 50 \%, 70 \%, 90 \%$, each of which was diluted using double distilled and deionized water) followed by two changes of acetone $100 \%$ (respectively of 10 and $30 \mathrm{~min}$ ) at room temperature and one change in $50 \%$ acetone epoxy resin for $1 \mathrm{~h}$. Finally, the samples were embedded in resin and cured at $60{ }^{\circ} \mathrm{C}$ for $24 \mathrm{~h}$. The obtained resin blocks were trimmed to expose the selected tissues and cut into thin sections (about $90 \mathrm{~nm}$ thick) with an ultramicrotome (Leica EM UC6) using a Diatome diamond knife. Single sections were positioned onto 50 mesh carbon coated copper grids and examined at $80-100 \mathrm{kV}$ with a JEOL JEM-1011 transmission electron microscope.

High angle annular dark field (HAADF) scanning transmission electron microscopy (STEM) analyses were carried out using a JEOL 2200FS microscope operating at $200 \mathrm{kV}$ and equipped with a field emission gun. The electron beam formed a $0.7 \mathrm{~nm}$ diameter probe, and this mode was used to collect EDX point spectra (Jeol Si(Li) EDS detector).

\subsection{Inductively coupled plasma-mass spectrometry analyses}

To assess AuNP distribution in Drosophila, we measured gold dosage by inductively coupled plasma-mass spectrometry (ICP-MS). Organisms nurtured for one month with food containing $15 \mathrm{~nm}$ AuNPs (380 pmol/L) were transferred in fresh normal food for two days to remove the previously ingested food containing AuNPs from the gastrointestinal tract. Subsequently, 10 flies were digested over night in an acidic solution consisting of $3 \mathrm{~mL}$ of $\mathrm{HNO}_{3} / \mathrm{H}_{2} \mathrm{O}_{2}$ (3:1), with a subsequent addition of $1 \mathrm{~mL}$ of $\mathrm{HCl}(37 \%)$. After $2 \mathrm{~h}$, samples were diluted with milliQ water to a final volume of $10 \mathrm{~mL}$ and analyzed. Flies nurtured with normal food were used as control.

\subsection{Statistical analyses}

All data in the figures are presented as mean \pm standard deviation (SD). For group comparison, we used twotailed Student's $t$-test. The survival distributions in control and experimental groups were also assessed in terms of significance using the non-parametric Mantel-Cox test. The effect of treatments on male and female fertility was evaluated by a two-tailed Student's $t$-test and by analyzing the significance of linear regression analysis.

\section{Results}

\subsection{Effects of AuNPs on Drosophila lifespan and reproductive performance}

The fruit fly Drosophila melanogaster is a well-established insect model for human diseases [24, 25] and represents a superb model for the study of genetics and cell biology, with distinct advantages for toxicology investigations [26, 27]. In order to investigate the possible effects of AuNPs in vivo, we performed lifespan studies on Drosophila. Consequently, we analyzed the lifespan of a fly population nurtured with $15 \mathrm{~nm}$ AuNP-treated food ( $380 \mathrm{pmol} / \mathrm{L})$ as compared to a control population bred with normal food. As a further control, we also tested the effect of treated food containing only the solvent of the AuNP suspension (namely, the medium in which the AuNPs were suspended, after removal of the AuNPs by centrifugation, hereafter referred to as supernatant, $\mathrm{SN}$ ). The $\mathrm{SN}$ was tested in order to exclude any possible toxic contribution from other components of the AuNP synthesis mixture, such as reaction precursors, surfactants and/or possible contaminants. The experiments were carried out by nurturing three Drosophila populations with the three different nutrition media during their entire life cycle, moving the flies into fresh vials every 4 days, and 
monitoring the number of survivors over time. The survival curves for the 3 populations are reported in Fig. 1. Surprisingly, we observed a very different behavior of the treated population with respect to the control, which shows the classical lifespan of Drosophila $[28,29]$. The survival curve of the AuNP-treated population exhibits a half-life $\left(\tau_{50 \%}\right)$ of ca. 2 weeks (14 days \pm 3 days), as opposed to the control curve showing the typical $\tau_{50 \%}$ of approximately 5 weeks (37 days \pm 3 days). This unexpected finding demonstrates that AuNPs strongly affect the life cycle of Drosophila, perturbing its normal lifespan and showing, therefore, an effect of generalized toxicity. Notably, the individuals treated with SN-modified food showed a behavior overlapping with the control.

In order to better clarify the effect of AuNPs on the life cycle of Drosophila, we investigated the influence of the nanoparticles on the reproductive performance of the flies. Drosophila organisms were treated with different doses of AuNPs (from 1.9 to $380 \mathrm{pmol} / \mathrm{L}$ ) for 10 days during the embryonic and larval development, and then adults were separated and subjected to fertility tests. Also in this case, AuNP-treated individuals were compared with both controls and SN-treated flies. As shown in Fig. 2, the reproductive performance of Drosophila is dramatically affected by the AuNPs. The fertility analyses revealed a clear concentrationdependent decrease of the reproductive performance, with fertility reductions approaching 50\% at the highest AuNP concentrations. Such a decrease is similar in the two sexes, suggesting a generalized mechanism of toxicity of AuNPs, which is not sex-linked.

\subsection{TEM analyses}

The presence and the distribution of the AuNPs in the Drosophila tissues were assessed by transmission electron microscopy (TEM). In these experiments we used individuals nurtured in treated food containing $380 \mathrm{pmol} / \mathrm{L}$ of AuNPs. Overall, the TEM analyses demonstrated that the ingestion of nanoparticles by Drosophila results in a quite homogenous distribution of the AuNPs throughout the deep layers of the enteric tissue, as well as in the reproductive organs. In the samples related to the digestive system (Fig. 3(a)), it is possible to observe a representative enterocyte section

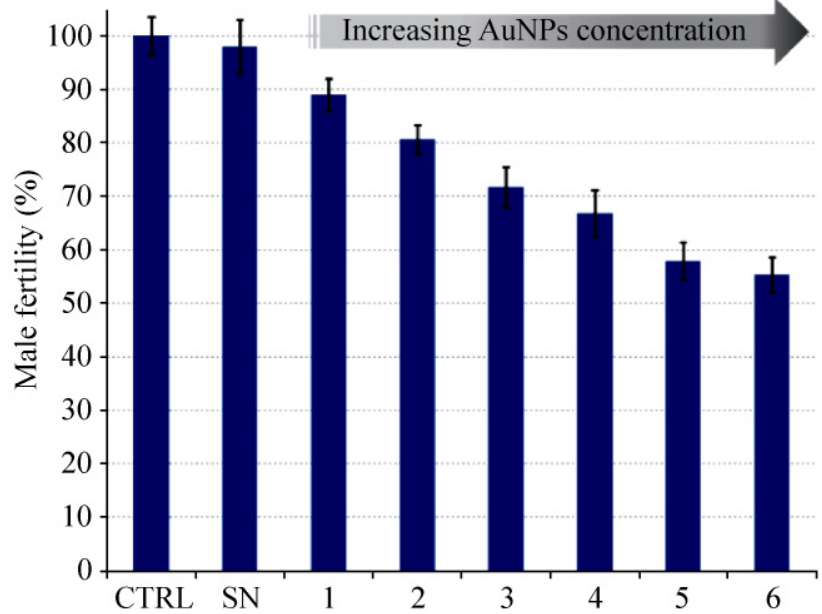

(a)

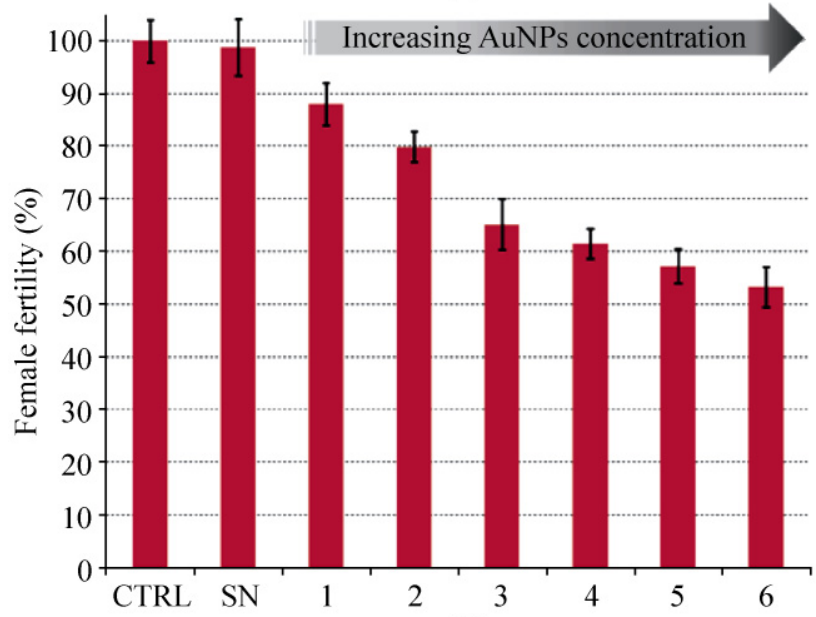

(b)

Figure 2 Male (a) and female (b) fertility tests of Drosophila melanogaster treated with increasing concentrations of AuNPs (1-6) as compared to control samples (CTRL) and supernatant treated samples (SN). AuNP concentrations of samples 1-6 were $1.9,3.8,19,38,190$, and $380 \mathrm{pmol} / \mathrm{L}$, respectively. Experimental points represent the average from 10 independent experiments and the error bars indicate the standard deviation. Data were statistically validated by linear regression analyses (Males: $p<0.05$; Females: $p<0.05$ )

in which many AuNPs are present, both as aggregates and monodispersed particles. In particular, most of AuNPs seem to be localized in the numerous electrontransparent vesicles of globular shape (i.e., endosomes), present near the lamellar structures of the rough endoplasmic reticulum (RER). The images from the isolated tissues of the reproductive organs revealed a different distribution of the AuNPs in the germinal lines of Drosophila (Figs. 3(b) and 3(c)). In particular, the
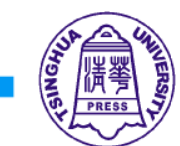

Springer 
sections of the ovariole (Fig. 3(b)) evidenced the presence of few metallic nanostructures in the cytoplasm (the black dots visible in the numerous mitochondria are calcium granules). On the other hand, in the testes, the AuNPs seem to be rather homogeneously distributed, being localized in the spermatocytes, but also at the perinuclear level in the spermatids (recognizable by the presence of the Nebenkern, composed of fused mitochondria) and in the flagella of mature motile sperm. In general, TEM analyses provided a direct observation of the localization of AuNPs in Drosophila tissues, which possibly correlates with the toxicity findings reported in Figs. 1 and 2. An additional representative image of the AuNP distribution is reported in Fig. S-2 (in the ESM). The presence of AuNPs in Drosophila tissues was also assessed by HAADF STEM, which demonstrated that the indicated dots are really gold nanostructures. Representative images are reported in Fig. S-3 (in the ESM).

\subsection{ICP-MS analyses}

The maximum dose of AuNPs administered to Drosophila in this work was $12 \mu \mathrm{g} / \mathrm{g}$ per day (corresponding to the $380 \mathrm{pmol} / \mathrm{L}$ AuNP concentration in food), assuming that the flies typically eat $1.5 \mu \mathrm{L} /$ day of food [22]. However, in order to assess the amount of AuNPs absorbed in Drosophila tissues, we performed ICP-MS analyses on the treated flies (after treatment for 1 month). We found the presence of $1.68 \mathrm{pg} \pm 0.14 \mathrm{pg}$ of gold per fly, corresponding to ca. 49000 AuNPs, indicating that only a small fraction of ingested particles enter the Drosophila tissues and causes the observed toxic effects (see also below).

\subsection{TUNEL assay}

The gastrointestinal tissue was further investigated in order to assess any occurrence of DNA damage in $15 \mathrm{~nm}$ AuNP-treated individuals (100 pmol/L). For this purpose, a specific fluorescence kit based on terminal deoxynucleotidyl transferase (TdT)-mediated TUNEL was used. Importantly, among the numerous nuclei displaying no DNA damage, we could observe a few cells in which DNA fragmentation was evident (Fig. 4). Such DNA damage was found to be distributed throughout the whole gastrointestinal tissue with no

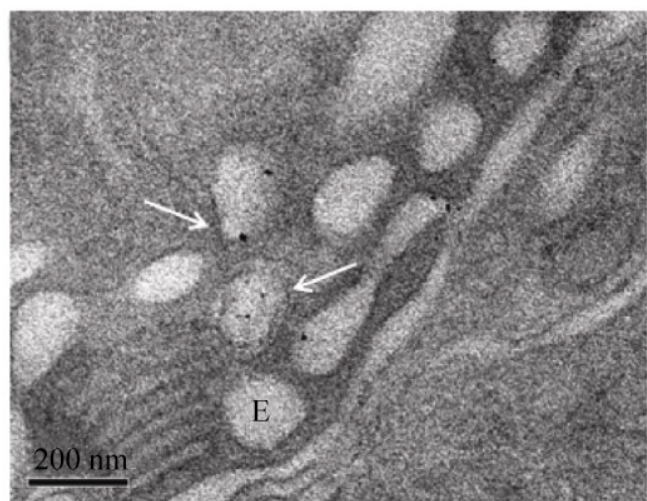

(a)

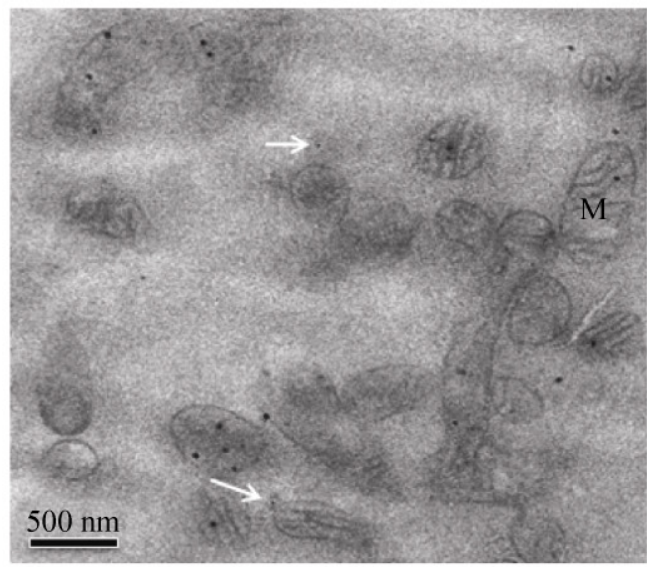

(b)

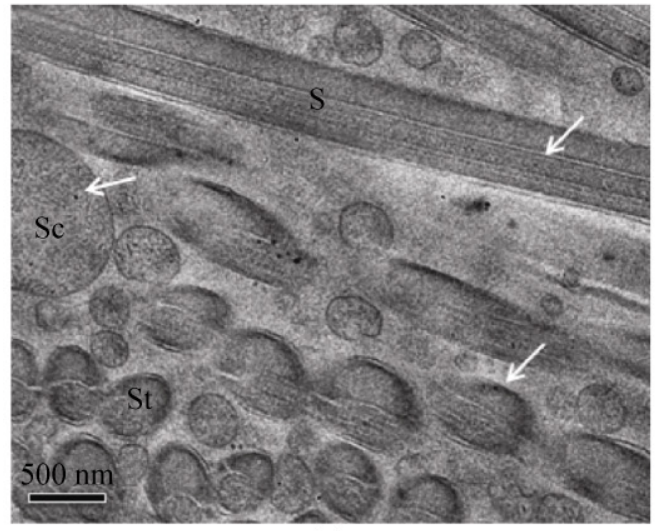

(c)

Figure 3 Representative TEM images of AuNP-treated (380 pmol/L) Drosophila. (a) Gastrointestinal tissue ("E" indicates an endosome; the RER is visible in the bottom-left part of the image). (b) Ovary ("M" indicates a mitochondrion; the black dots localized in the mitochondria are calcium granules evidenced by osmium fixation). (c) Testes ("Sc" indicates a spermatocyte, "St" indicates a spermatid, and "S" indicates the flagellum of a mature motile sperm; the black dots localized in the central part of the image are due to osmium fixation). The white arrows indicate some AuNPs found in the tissues. These TEM sections, particularly rich in AuNPs, have been selected to illustrate the typical distribution of the AuNPs in the Drosophila tissues, but do not represent the "average" AuNP concentration in the fly tissues 


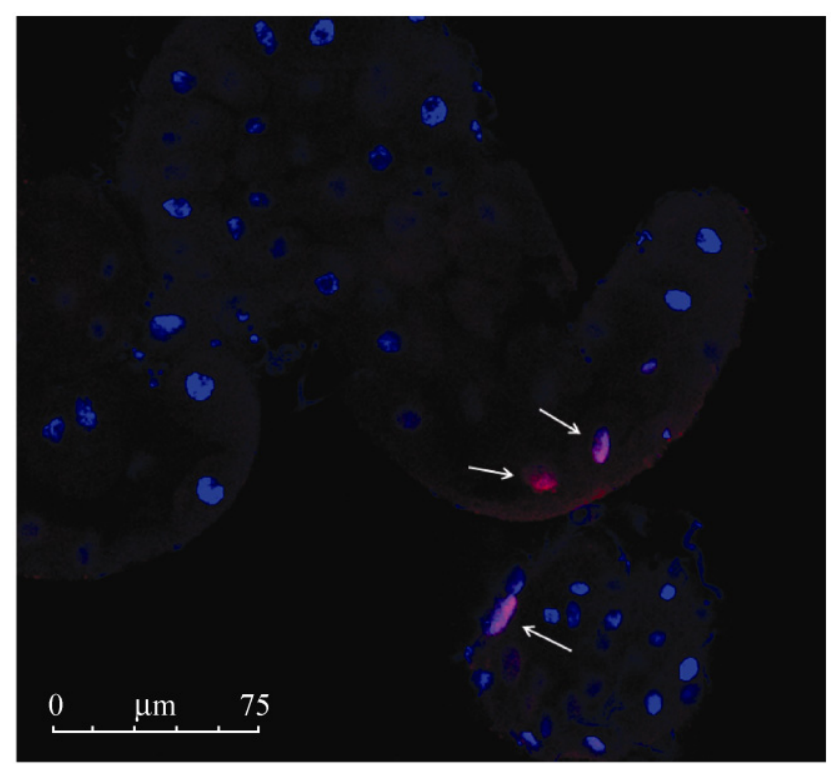

Figure 4 Representative confocal microscopy image of Drosophila midgut in flies treated with $15 \mathrm{~nm}$ AuNPs (100 pmol/L). Nuclei are stained with Hoechst 33342 (blue) while cells containing DNA strand nicks are detected by TUNEL assay and fluoresce red (highlighted by the white arrows)

apparent localization into specific regions. Several midgut samples were analyzed with this technique and the observation of DNA nicks in enterocytes was highly reproducible. On the contrary, in SN-treated samples as well as in control flies, weak fluorescent signals related to DNA strand nicks were typically detected. A quantitative analysis of the TUNEL assay revealed an occurrence of DNA damage of ca. $8 \%$ $(8.15 \% \pm 2.46 \%)$ in AuNP-treated flies, while in the control and SN-treated samples DNA fragmentation was less than $1 \%(0.84 \% \pm 0.43 \%)$ (Student's $t$-test $p$ $<0.001)$. This experimental evidence indicates a clear toxic effect of AuNPs on the gastrointestinal tissue. The localization of the $15 \mathrm{~nm}$ AuNPs in the enterocytes evidenced by TEM analyses (Fig. 3(a)) strongly suggests an indirect effect of the nanomaterials in causing DNA damage. Further analyses are required to better understand this point, but the observed fragmentation is very likely to be mediated by oxidative stress and/or related to early stage apoptosis.

\subsection{Expression of heat-shock-protein-70kDa}

In order to support the hypothesis of generalized toxicity elicited by the AuNPs in Drosophila, we performed molecular tests to evaluate the genetic expression of $h s p 70$ by means of quantitative real-time PCR (qRT-PCR). Hsp70 is one of the most conserved genes in the family of heat shock proteins, and it is a well established marker of stress, since it is the first to be overexpressed in Drosophila upon chemical, physical or physiological stress [30-33]. The experiments were performed by using mRNA extracted from AuNPtreated individuals as compared to flies nurtured with normal and SN-treated food. The results obtained by qRT-PCR are reported in Fig. 5. Interestingly, it is possible to observe in AuNP-treated organisms an overexpression of $h s p 70$ as high as $272 \%$ with respect to the control (the results from the SN-treated flies were comparable to the control). Such an increase of hsp70 expression represents a clear indication of generalized toxicity induced by the AuNPs in the whole organism. Moreover, this evidence strongly supports the data of premature mortality observed in the lifespan experiments and the reduced reproductive performance found in the fertility tests [34-36].

\section{Discussion}

We have observed that citrate-capped AuNPs induce significant toxicity in the model animal Drosophila melanogaster upon ingestion, manifested by the decrease

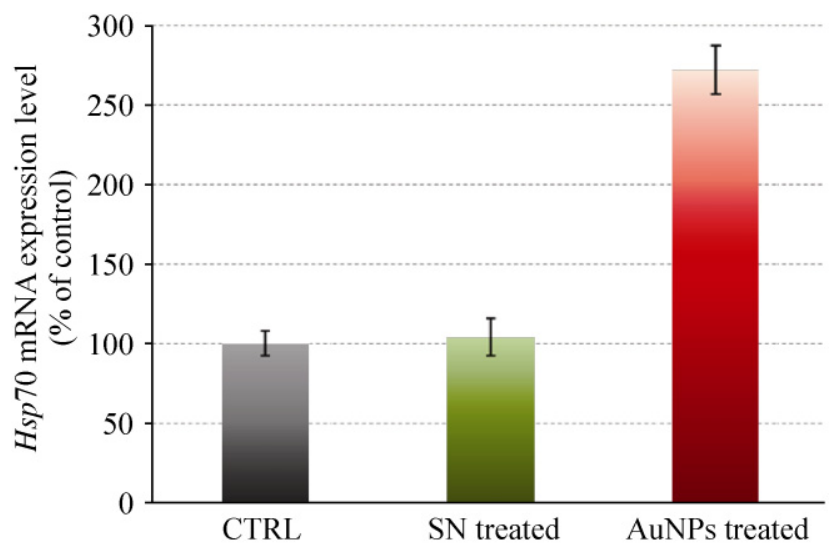

Figure $5 H s p 70$ expression levels in AuNP-treated (380 pmol/L) flies as compared to control and SN-treated individuals. Experimental points represent the average from 5 independent experiments and the error bars indicate the standard deviation (significance of different expression levels was evaluated by Student's $t$-test giving $p<0.01$, AuNP treatment compared to the control; $p<0.01$, AuNPs compared to the $\mathrm{SN} ; p>0.05$, control compared to the $\mathrm{SN}$ )

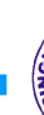

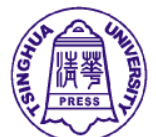

算 Springer 
of both lifespan and fertility, presence of DNA fragmentation in the gastrointestinal tissue, and by the remarkable overexpression of the stress marker $h s p 70$. The detailed mechanisms underlying the toxicity processes are currently unknown and require further extensive investigations. A key role, however, is likely to be played by the peculiar physicochemical features of the material at the nanoscale, such as the increased surface-to-volume ratio [1] and the high surface area and reactivity "seen" by the biological systems. The increased surface-to-volume $(S / V)$ ratio, growing with $1 / R$ ( $R$ being the NP radius), causes small NPs to have a large amount of states on the surface available for chemical reactions, as opposed to the bulk case. Consequently, although bulk gold is known to be inert, nanoscale gold structures may be extremely effective oxidation catalysts [10], that can perturb metabolic processes in a cell. In particular, it is possible to envisage a role of the NPs in increasing the oxidative stress, leading to reactive oxygen species (ROS) generation and consequent membrane, protein and DNA damage (as also evidenced by TUNEL assay in the midgut), that possibly accounts for the observed fertility decrease. On the other hand, the localization of NPs in the different tissues might imply several specific cellular processes involving organelles and/or specific pathways, besides the mechanisms of generalized toxicity, also revealed by the $h s p 70$ overexpression.

A detailed study of the size-dependence (R-dependence) of the toxicity of AuNPs in vivo, as well as the influence of the size and surface chemistry of the AuNPs in the localization, intracellular fate and toxicological effects/pathways is underway in our laboratory. In any case, the experimental evidence of high in vivo toxicity of a nanoscale material, which is widely considered to be safe and biocompatible in its bulk form, has several implications and opens up important questions (although it is possible that different surface coatings of AuNPs, e.g., pegylation, may partly/significantly reduce their in vivo toxicity). In our opinion, a higher awareness of such potential problems along with a serious strategy for risk assessment and nanosafety are strongly required in the next few years, as several nanomaterials are being increasingly exploited in both commercial goods and novel applications.

\section{Acknowledgements}

The authors gratefully acknowledge S. Shiv Shankar for useful discussions, and B. Antonazzo, V. Fiorelli and M. Malerba for the expert technical assistance.

Electronic Supplementary Material: Supplementary material (characterization of AuNPs, additional TEM images of the distribution of $15 \mathrm{~nm}$ AuNPs in the Drosophila tissues, and STEM imaging with EDS analyses) is available in the online version of this article at http://dx.doi.org/10.1007/s12274-011-0095-z and is accessible free of charge.

\section{References}

[1] Nel, A.; Xia, T.; Madler, L.; Li, N. Toxic potential of materials at the nanolevel. Science 2006, 311, 622-627.

[2] Nel, A. E.; Madler, L.; Velegol, D.; Xia, T.; Hoek, E. M. V.; Somasundaran, P.; Klaessig, F.; Castranova, V.; Thompson, M. Understanding biophysicochemical interactions at the nano-bio interface. Nat. Mater. 2009, 8, 543-557.

[3] Oberdorster, G.; Oberdorster, E.; Oberdorster, J. Nanotoxicology: An emerging discipline evolving from studies of ultrafine particles. Environ. Health Perspect. 2005, 113, 823-839.

[4] Dobrovolskaia, M. A.; Germolec, D. R.; Weaver, J. L. Evaluation of nanoparticle immunotoxicity. Nat. Nanotechnol. 2009, 4, 411-414.

[5] Maynard, A. D.; Aitken, R. J.; Butz, T.; Colvin, V.; Donaldson, K.; Oberdorster, G.; Philbert, M. A.; Ryan, J.; Seaton, A.; Stone, V.; Tinkle, S. S.; Tran, .; Walker, N. J.; Warheit, D. B. Safe handling of nanotechnology. Nature 2006, 444, 267-269.

[6] Poland, C. A.; Duffin, R.; Kinloch, I.; Maynard, A.; Wallace, W. A. H.; Seaton, A.; Stone, V.; Brown, S.; MacNee, W.; Donaldson, K. Carbon nanotubes introduced into the abdominal cavity of mice show asbestos-like pathogenicity in a pilot study. Nat. Nanotechnol. 2008, 3, 423-428.

[7] Mortensen, L. J.; Oberdorster, G.; Pentland, A. P.; Delouise, L. A. In vivo skin penetration of quantum dot nanoparticles in the murine model: The effect of UVR. Nano Lett. 2008, 8 , 2779-2787.

[8] AshaRani, P. V.; Mun, G. L. K.; Hande, M. P.; Valiyaveettil, S. Cytotoxicity and genotoxicity of silver nanoparticles in human cells. ACS Nano 2009, 3, 279-290.

[9] Napierska, D.; Thomassen, L. C.; Rabolli, V.; Lison, D.; Gonzalez, L.; Kirsch-Volders, M.; Martens, J. A.; Hoet, P. H. Size-dependent cytotoxicity of monodisperse silica nanoparticles in human endothelial cells. Small 2009, 5, 846-853. 
[10] Auffan, M.; Rose, J.; Bottero, J. Y.; Lowry, G. V.; Jolivet, J. P.; Wiesner, M. R. Towards a definition of inorganic nanoparticles from an environmental, health and safety perspective. Nat. Nanotechnol. 2009, 4, 634-641.

[11] Alkilany, A. M.; Nagaria, P. K.; Hexel, C. R.; Shaw, T. J.; Murphy, C. J.; Wyatt, M. D. Cellular uptake and cytotoxicity of gold nanorods: Molecular origin of cytotoxicity and surface effects. Small 2009, 5, 701-708.

[12] Shukla, R.; Bansal, V.; Chaudhary, M.; Basu, A.; Bhonde, R. R.; Sastry, M. Biocompatibility of gold nanoparticles and their endocytotic fate inside the cellular compartment: A microscopic overview. Langmuir 2005, 21, 10644-10654.

[13] Connor, E. E.; Mwamuka, J.; Gole, A.; Murphy, C. J.; Wyatt, M. D. Gold nanoparticles are taken up by human cells but do not cause acute cytotoxicity. Small 2005, 1, 325-327.

[14] Murphy, C. J.; Gole, A. M.; Stone, J. W.; Sisco, P. N.; Alkilany, A. M.; Goldsmith, E. C.; Baxter, S. C. Gold nanoparticles in biology: Beyond toxicity to cellular imaging. Acc. Chem. Res. 2008, 41, 1721-1730.

[15] Pernodet, N.; Fang, X.; Sun, Y.; Bakhtina, A.; Ramakrishnan, A.; Sokolov, J.; Ulman, A.; Rafailovich, M. Adverse effects of citrate/gold nanoparticles on human dermal fibroblasts. Small 2006, 2, 766-773.

[16] Pan, Y.; Neuss, S.; Leifert, A.; Fischler, M.; Wen, F.; Simon, U.; Schmid, G.; Brandau, W.; Jahnen-Dechent, W. Sizedependent cytotoxicity of gold nanoparticles. Small 2007, 3, 1941-1949.

[17] Hauck, T. S.; Ghazani, A. A.; Chan, W. C. W. Assessing the effect of surface chemistry on gold nanorod uptake, toxicity, and gene expression in mammalian cells. Small 2008, 4, 153-159.

[18] Khan, J. A.; Pillai, B.; Das, T. K.; Singh, Y.; Maiti, S. Molecular effects of uptake of gold nanoparticles in HeLa cells. ChemBioChem 2007, 8, 1237-1240.

[19] Li, J. J.; Zou, L.; Hartono, D.; Ong, C. N.; Bay, B. H.; Yung, L. Y. L. Gold nanoparticles induce oxidative damage in lung fibroblasts in vitro. Adv. Mater. 2008, 20, 138-142.

[20] Turkevich, J.; Stevenson, P. C.; Hillier, J. A study of the nucleation and growth processes in the synthesis of colloidal gold. Discuss. Faraday Soc. 1951, 11, 55-75.

[21] Frens, G. Controlled nucleation for the regulation of the particle size in monodisperse gold suspensions. Nat. Phys. Sci. 1973, 241, 20-22.

[22] Ja, W. W.; Carvalho, G. B.; Mak, E. M.; de la Rosa, N. N.; Fang, A. Y.; Liong, J. C.; Brummel, T.; Benzer, S. Prandiology of Drosophila and the CAFE assay. Proc. Natl. Acad. Sci. USA 2007, 104, 8253-8256.

[23] Gayathri, M. V.; Krishnamurthy, N. B. Studies on the toxicity of the mercurial fungicide Agallol 3 in Drosophila melanogaster. Environ. Res. 1981, 24, 89-95.

[24] Auluck, P. K.; Chan, H. Y. E.; Trojanowski, J. Q.; Lee, V. M. Y.; Bonini, N. M. Chaperone suppression of alpha-synuclein toxicity in a Drosophila model for Parkinson's disease. Science 2002, 295, 865-868.

[25] Kazantsev, A.; Walker, H. A.; Slepko, N.; Bear, J. E.; Preisinger, E.; Steffan, J. S.; Zhu, Y. Z.; Gertler, F. B.; Housman, D. E.; Marsh, J. L.; Thompson, L. M. A bivalent Huntingtin binding peptide suppresses polyglutamine aggregation and pathogenesis in Drosophila. Nat. Genet. 2002, 30, 367-376.

[26] Benford, D. J.; Hanley, A. B.; Bottrill, K.; Oehlschlager, S.; Balls, M.; Branca, F.; Castegnaro, J. J.; Descotes, J.; Hemminiki, K.; Lindsay, D.; Schiliter, B. Biomarkers as predictive tools in toxicity testing-The report and recommendations of ECVAM Workshop 40. ATLA-Alternatives to Laboratory Animals 2000, 28, 119-131.

[27] Ahamed, M.; Posgai, R.; Gorey, T. J.; Nielsen, M.; Hussain, S. M.; Rowe, J. J. Silver nanoparticles induced heat shock protein 70, oxidative stress and apoptosis in Drosophila melanogaster. Toxicol. Appl. Pharmacol., 242, 263-269.

[28] Lin, Y. J.; Seroude, L.; Benzer, S. Extended life-span and stress resistance in the Drosophila mutant methuselah. Science 1998, 282, 943-946.

[29] Zid, B. M.; Rogers, A. N.; Katewa, S. D.; Vargas, M. A.; Kolipinski, M. C.; Lu, T. A.; Benzer, S.; Kapahi, P. 4E-BP extends lifespan upon dietary restriction by enhancing mitochondrial activity in Drosophila. Cell 2009, 139, 149-160.

[30] Feder, J. H.; Rossi, J. M.; Solomon, J.; Solomon, N.; Lindquist, S. The consequences of expressing hsp70 in Drosophila cells at normal temperatures. Genes Dev. 1992, 6, 1402-1413.

[31] Singh, M. P.; Reddy, M. M. K.; Mathur, N.; Saxena, D. K.; Chowdhuri, D. K. Induction of hsp70, hsp60, hsp83 and hsp26 and oxidative stress markers in benzene, toluene and xylene exposed Drosophila melanogaster: Role of ROS generation. Toxicol. Appl. Pharmacol. 2009, 235, 226-243.

[32] Morimoto, R. I. Cells in stress: Transcriptional activation of heat shock genes. Science 1993, 259, 1409-1410.

[33] Pockley, A. G. Heat shock proteins as regulators of the immune response. Lancet 2003, 362, 469-476.

[34] Tatar, M.; Khazaeli, A. A.; Curtsinger, J. W. Chaperoning extended life. Nature 1997, 390, 30.

[35] Silbermann, R.; Tatar, M. Reproductive costs of heat shock protein in transgenic Drosophila melanogaster. Evolution 2000, 54, 2038-2045.

[36] Partridge, L.; Gems, D. Mechanisms of ageing: Public or private? Nat. Rev. Genet. 2002, 3, 165-175. 